\title{
Systematic Review \\ Regional Odontodysplasia: A Systematic Review of Case Reports
}

\author{
Kacper Nijakowski ${ }^{1, *++}$ (D), Patryk Woś ${ }^{2,+}$ and Anna Surdacka ${ }^{1}$ (D) \\ 1 Department of Conservative Dentistry and Endodontics, Poznan University of Medical Sciences, \\ 60-812 Poznan, Poland; annasurd@ump.edu.pl \\ 2 Student's Scientific Group, Department of Conservative Dentistry and Endodontics, Poznan University of \\ Medical Sciences, 60-812 Poznan, Poland; patrykwos4@gmail.com \\ * Correspondence: kacpernijakowski@ump.edu.pl \\ + These authors contributed equally to this work.
}

Citation: Nijakowski, K.; Woś, P.; Surdacka, A. Regional Odontodysplasia: A Systematic Review of Case Reports. Int. J. Environ. Res. Public Health 2022, 19, 1683. https://doi.org/10.3390/ ijerph19031683

Academic Editors: Joanna Baginska and Ewa Joanna Rodakowska

Received: 13 December 2021

Accepted: 30 January 2022

Published: 1 February 2022

Publisher's Note: MDPI stays neutral with regard to jurisdictional claims in published maps and institutional affiliations.

Copyright: (c) 2022 by the authors. Licensee MDPI, Basel, Switzerland. This article is an open access article distributed under the terms and conditions of the Creative Commons Attribution (CC BY) license (https:// creativecommons.org/licenses/by/ $4.0 /)$.

\begin{abstract}
Regional odontodysplasia is a rare developmental disorder characterised by hypoplasia and hypomineralisation of enamel and dentin. Our systematic review aimed to organise the knowledge on localisation, symptomatology and treatment methods in patients with regional odontodysplasia based on case reports published in the databases PubMed, Scopus and Web of Science. Case reports were described in 28 different countries, considering 180 patients (including 91 females). Regional odontodysplasia occurs mainly in both deciduous and permanent dentition (66.1\%). The affected teeth were observed more frequently in the maxilla $(70.0 \%)$, especially on the left side $(45.6 \%)$. The most common reported symptoms were ghost teeth, poorly developed buds, yellowish-brown colour of crowns and delayed eruption of permanent teeth in affected quadrants. The most popular treatment method was surgical treatment $(78.6 \%)$ with subsequent prosthetic therapy $(34.6 \%)$. Based on the review of cases, pathognomonic clinical and radiological signs can be found, however, it is difficult to reach a consensus on the choice of treatment method.
\end{abstract}

Keywords: regional odontodysplasia; dental anomaly; ghost teeth; systematic review; case reports

\section{Introduction}

Regional odontodysplasia ( $\mathrm{RO}$ ) is a rare developmental anomaly characterised by hypoplasia and hypocalcification of dental hard tissues. The condition was probably first described by Hitchin in 1934, but the term was first used in 1963 by Zegarelli et al. [1]. Among three case reports, most of the anomalous teeth were unerupted and intraosseously located, as well as the erupted teeth were also poorly developed. It was observed that the calcification of enamel and dentin had appeared to be severely deficient in both quality and quantity. The prefix "regional" was added by Pindborg in 1970, considering its localised nature [2,3]. It usually affects one quadrant; however, cases only affecting one tooth or generalised have been reported in the literature [4].

The altered teeth are commonly yellow or brown and show abnormal morphology. Most patients suffer from recurrent purulent inflammations or tooth mobility. Wide pulp chambers, open apices and blurred demarcation in the dentino-enamel junction are the main radiological features distinctive of $\mathrm{RO}$ [5]. The disorder involves both primary and permanent dentitions in the majority of the cases. Histologically, both enamel and dentin appear hypomineralised with poorly organised dentinal tubules and enamel prisms. The pulp chambers very often contain calcifications in the coronary area [6].

The aetiology remains still unknown. Several factors have been suggested in the literature, such as teratogenic medications used during pregnancy, latent viruses, circulatory disorders, radiation, hyperpyrexia accompanying severe childhood disease, nutritional deficiencies, Rh disease, local trauma or ischemia [7]. Both trauma and circulatory disorders seem to be relevant, as they have appeared several times as possible factors among the case 
reports [8-11]. There are some examples of hereditary prevalence suggesting genetic causes in some of the patients. Koskinen et al. [12] described a 6.5-year-old girl, whose mother and two sisters (all of the siblings) had partially missing teeth with no other symptoms. On the contrary, there are also cases highlighting the absence of a positive familial history in the affected patients $[13,14]$. Even though many authors suggest the potential cause, no general conclusions have been made.

There are relatively few case reports of regional odontodysplasia in the literature, e.g., in Poland we are the first to describe this anomaly [15]. A 5-year-old male patient was referred to our department. The main complaint was recurrent purulent inflammations of deciduous teeth in the third quadrant. The affected teeth differed from the healthy because of their yellowish-brown discolouration and tendency to crumble rapidly. The pregnancy, medical and family histories were unremarkable. Other teeth did not present any pathology. Based on the panoramic radiograph taken three months before the appointment, the mandibular left deciduous teeth and the follicles of succedaneous permanent teeth demonstrated a "ghost-like" appearance (Figure 1). Moreover, the underdevelopment of the left side of the mandible and the slight shift of the midline to the left were noticeable. The periapical radiographs presented blurred demarcation between thin layers of enamel and dentin in the affected teeth. Additionally, wide pulp chambers with open apices were visible for "ghost teeth". The proposed treatment was the surgical removal of the residual roots of the primary mandibular left first molar. A week later, on a follow-up visit, the active fistula and increased mobility were observed in the primary left incisors. Therefore, they were extracted too. After a month, it was decided to remove the last deciduous tooth in this quadrant because of severe pain during biting. Due to the early loss of affected primary teeth, the patient was referred for a removable acrylic partial denture. Two years after the first visit, the follow-up panoramic radiograph presented slight progress in the development of the permanent teeth in the affected region.

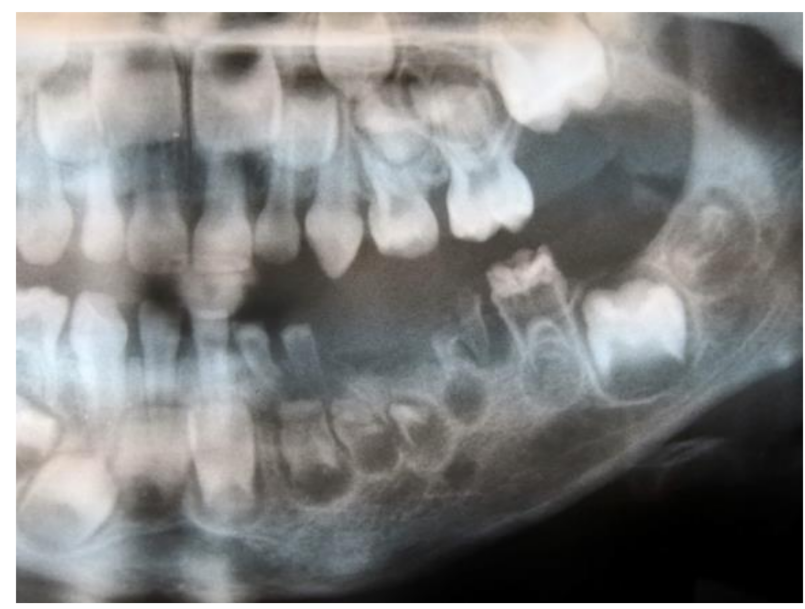

Figure 1. Fragment of the panoramic radiograph showing "ghost teeth" (in the mandibular left quadrant) characteristic of regional odontodysplasia-personal collection (K.N., Poland, 2019).

Our systematic review aimed to organise the existing knowledge on localisation, symptomatology and treatment methods in patients with regional odontodysplasia based on published case reports.

\section{Materials and Methods}

This systematic review was conducted up to 1 September 2021, according to the Preferred Reporting Items for Systematic Reviews and Meta-Analyses (PRISMA) statement guidelines [16], using the databases PubMed, Scopus and Web of Science. The search formula included "odontodysplasia" or "regional odontodysplasia" or "ghost teeth" as $\mathrm{MeSH}$ (medical subject headings) terms combined in PubMed Advanced Search Builder. In 
other databases, the same keywords were used (in Scopus as index terms and in Web of Science as author keywords or keywords plus).

Records were screened by the title, abstract and full text by two independent investigators. Studies included in this review matched all the predefined criteria according to the PICOS ("Population", "Intervention", "Comparison", "Outcomes", "Study design")—Table 1. A detailed search flowchart is presented in Figure 2 (in Section 3).

Table 1. Inclusion and exclusion criteria according to the PICOS.

\begin{tabular}{ccc}
\hline Parameter & Inclusion Criteria & Exclusion Criteria \\
\hline Population & $\begin{array}{c}\text { patients with regional } \\
\text { odontodysplasia-aged from 0 to } 99 \\
\text { years, both sexes } \\
\text { not applicable } \\
\text { Comparison }\end{array}$ & $\begin{array}{c}\text { patients with other developmental } \\
\text { dental anomalies }\end{array}$ \\
Outcomes & $\begin{array}{c}\text { clinical description with radiological } \\
\text { features and/or histopathological } \\
\text { analyses }\end{array}$ & $\begin{array}{c}\text { only histopathological or another } \\
\text { analysis without clinical description } \\
\text { literature reviews, expert opinion, }\end{array}$ \\
Study design & case reports & $\begin{array}{c}\text { letters to editor, conference reports, } \\
\text { case-control, cohort and } \\
\text { cross-sectional studies }\end{array}$ \\
& published up to September 2021 & not published in English
\end{tabular}

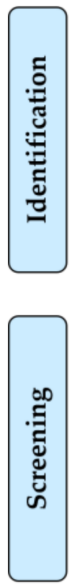

Records identified through PubMed database advanced searching $(n=347)$

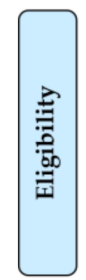

Additional full-text records identified through Google Scholar $(n=15)$

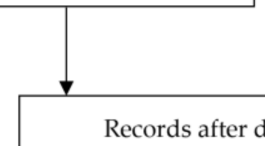

$(n=361)$

Additional records identified through other sources: Scopus $(n=343)$ and Web of Science $(n=119)$
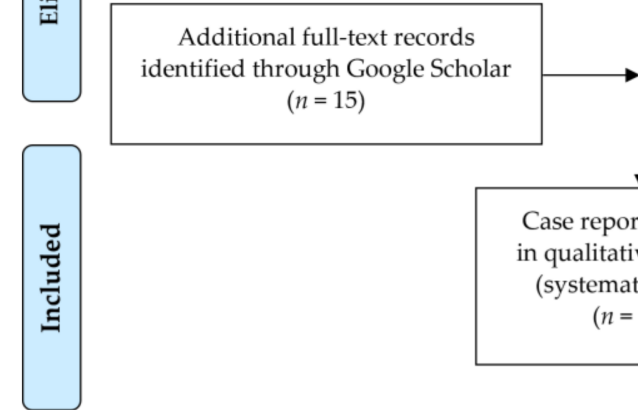

$$
\begin{gathered}
\text { Case reports included } \\
\text { in qualitative synthesis } \\
\text { (systematic review) }
\end{gathered}
$$
$(n=130)$

Figure 2. PRISMA flow diagram presenting the detailed search strategy.

The critical appraisal of case reports was assessed according to the JBI Critical Appraisal Tool issued by the Faculty of Health and Medical Sciences at the University of 
Adelaide, South Australia. These questionnaires were answered by two independent investigators, and disagreements were resolved by discussion between them. Due to the rarity of the condition, the decision was made to include all papers dealing with regional odontodysplasia and containing basic patient information, including necessarily the location of the condition. Among the included papers, one report was without the gender of the patient, 21 patients without a detailed macroscopic image of the affected teeth, 14 patients without an attached radiograph, and 21 patients without the treatment method used (with only diagnostic description of the patients). Additionally, additional histopathologic findings were presented in 80 patients.

All included studies have a 4th level of evidence, according to the classification of the Oxford Center for Evidence-Based Medicine levels for diagnosis [17].

\section{Results}

In this systematic review, 130 papers following the search criteria were included (the list is attached as Supplementary Materials). Case reports were described in 28 different countries, considering a total of 180 patients (including 91 females). Among them, 74 contained long-term descriptions of clinical observations. Figure 2 shows the detailed selection strategy of the articles. The inclusion and exclusion criteria are presented in Table 1 (in Section 2).

Regardless of the sex of the patients, regional odontodysplasia occurs in both deciduous and permanent dentition $(66.1 \%)$. The affected teeth were observed more frequently in the maxilla $(70.0 \%)$, especially on the left side (45.6\%). Generalised odontodysplasia was described in only 10 patients (5.5\%). The distribution of locations in the included cases is presented in Table 2.

Table 2. Location of teeth affected by regional odontodysplasia (\%).

\begin{tabular}{|c|c|c|c|c|c|}
\hline & & $\begin{array}{c}\text { All } \\
n=180\end{array}$ & $\begin{array}{c}\text { Male } \\
n=88\end{array}$ & $\begin{array}{c}\text { Female } \\
n=91\end{array}$ & $p$-Value \\
\hline \multirow{3}{*}{ dentition } & primary & 5.0 & 3.4 & 6.6 & \multirow{3}{*}{0.479} \\
\hline & secondary & 28.9 & 29.5 & 27.5 & \\
\hline & both & 66.1 & 67.1 & 65.9 & \\
\hline \multirow{3}{*}{ jaw } & maxilla & 61.1 & 58.0 & 63.7 & \multirow{3}{*}{0.680} \\
\hline & mandible & 30.0 & 34.0 & 26.4 & \\
\hline & both & 8.9 & 8.0 & 9.9 & \\
\hline \multirow{4}{*}{ quadrants } & 1st & 44.4 & 47.7 & 40.7 & \multirow{4}{*}{$\begin{array}{c}0.281 \\
0.048 * \\
0.665 \\
0.497\end{array}$} \\
\hline & 2nd & 45.6 & 37.5 & 53.8 & \\
\hline & $3 r d$ & 25.6 & 27.3 & 24.2 & \\
\hline & 4 th & 28.9 & 26.1 & 31.9 & \\
\hline \multirow{11}{*}{ affected region } & left side of maxilla & 25.0 & 18.2 & 31.9 & \multirow{11}{*}{0.362} \\
\hline & right side of maxilla & 21.7 & 23.8 & 18.7 & \\
\hline & left side of mandible & 10.0 & 15.9 & 4.4 & \\
\hline & right side of mandible & 11.7 & 12.5 & 11.0 & \\
\hline & bilateral maxilla & 14.4 & 15.9 & 13.2 & \\
\hline & bilateral mandible & 8.3 & 5.7 & 11.0 & \\
\hline & ipsilateral left quadrants & 0.0 & 0.0 & 0.0 & \\
\hline & ipsilateral right quadrants & 1.7 & 2.3 & 1.1 & \\
\hline & contralateral quadrants & 0.0 & 0.0 & 0.0 & \\
\hline & without one quadrant & 1.7 & 2.3 & 1.1 & \\
\hline & generalised & 5.5 & 3.4 & 7.6 & \\
\hline
\end{tabular}

* $p$-value $<0.05$ for the Chi-square test.

In the clinical examination, the most common findings were yellowish-brown colour of crowns (90.6\%) and delayed eruption of permanent teeth (90.6\%). Moreover, the radiographs practically always showed ghost teeth $(100.0 \%)$ and poorly developed buds $(92.2 \%)$ in affected quadrants. The presence of other features is shown in Table 3. Taking into account gender, in female patients swelling was observed significantly more often, as 
well as local pain and mobility differed closely to statistical significance. Radiologically, no differences in the frequency of symptoms in patients of different sexes were noted.

Table 3. Clinical and radiological features in patients with regional odontodysplasia (\%).

\begin{tabular}{|c|c|c|c|c|}
\hline Clinical Features & $\begin{array}{c}\text { All } \\
n=159\end{array}$ & $\begin{array}{l}\text { Male } \\
n=76\end{array}$ & $\begin{array}{c}\text { Female } \\
n=83\end{array}$ & $p$-Value \\
\hline yellowish-brown colour & 90.6 & 89.5 & 91.6 & 0.652 \\
\hline gingival swelling & 67.3 & 55.3 & 78.3 & $0.002 *$ \\
\hline $\begin{array}{l}\text { underdeveloped one side of } \\
\text { maxilla/mandible }\end{array}$ & 17.6 & 21.1 & 14.5 & 0.276 \\
\hline active fistulas & 18.9 & 18.4 & 19.3 & 0.890 \\
\hline tooth mobility & 41.5 & 34.2 & 48.2 & 0.074 \\
\hline early loss of primary teeth & 47.8 & 50.0 & 45.8 & 0.595 \\
\hline delayed tooth eruption & 90.6 & 93.4 & 88.0 & 0.239 \\
\hline local pain & 44.7 & 36.8 & 51.8 & 0.058 \\
\hline Radiological features & $\begin{array}{c}\text { All } \\
n=166\end{array}$ & $\begin{array}{l}\text { Male } \\
n=79\end{array}$ & $\begin{array}{c}\text { Female } \\
n=87\end{array}$ & $p$-Value \\
\hline ghost teeth & 100 & 100 & 100 & - \\
\hline poorly developed buds & 92.2 & 91.1 & 93.1 & 0.638 \\
\hline periapical lesions & 39.2 & 40.5 & 37.9 & 0.734 \\
\hline
\end{tabular}

* $p$-value $<0.05$ for the Chi-square test.

Based on Table 4, the most popular treatment method used in patients with odontodysplasia was surgical treatment (78.6\%) accompanied by prosthetic treatment (34.6\%). Similarly, according to gender, no significant differences were found in the distributions of individual treatment methods.

Table 4. Treatment methods in patients with regional odontodysplasia (\%).

\begin{tabular}{ccccc}
\hline Treatment Method & $\begin{array}{c}\text { All } \\
\boldsymbol{n}=\mathbf{1 5 9}\end{array}$ & $\begin{array}{c}\text { Male } \\
\boldsymbol{n}=\mathbf{7 5}\end{array}$ & $\begin{array}{c}\text { Female } \\
\boldsymbol{n}=\mathbf{8 3}\end{array}$ & $\boldsymbol{p}$-Value \\
\hline conservative restoration & 14.5 & 18.7 & 10.8 & 0.164 \\
steel crown & 5.7 & 8.0 & 3.6 & 0.235 \\
pulpotomy/RCT & 2.5 & 4.0 & 1.2 & 0.264 \\
tooth extraction & 78.6 & 74.7 & 83.1 & 0.191 \\
acrylic partial denture & 34.6 & 40.0 & 30.1 & 0.193 \\
orthodontic treatment & 16.4 & 21.3 & 12.0 & 0.116 \\
implantoprosthetic treatment & 7.5 & 6.7 & 8.4 & 0.676 \\
autotransplantation & 1.9 & 1.3 & 2.4 & 0.621 \\
\hline
\end{tabular}

Table 5 shows the histopathological changes found in the involved teeth. Irregular calcification of interglobular dentin $(92.5 \%)$ and hypoplasia or hypomineralisation of enamel $(77.5 \%)$ were the most frequently observed.

Table 5. Histopathological features in patients with regional odontodysplasia (\%).

\begin{tabular}{cc}
\hline Histopathological Features & $\mathbf{n = 8 0}$ \\
\hline hypoplastic and hypomineralised enamel & 77.5 \\
irregular calcification of dentin & 92.5 \\
irregular dentino-enamel junction & 28.8 \\
pulpal calcifications & 45.0 \\
dislocated cementum & 8.8 \\
\hline
\end{tabular}

\section{Discussion}

Regional odontodysplasia is a condition that we still know very little about. There are only suggestions of possible aetiology, and therefore there is no possibility of preventing its 
emergence. The purpose of this review was to collect data from all available case reports, including those reported in the recent past and draw conclusions based on the results compared to similar works from the past.

As seen in the table, the condition appears more often in the maxilla $(61.1 \%)$ than in the mandible (30\%); the remaining cases had symptoms both in the upper and lower jaws $(8.9 \%)$. The predilection is similar to what was found by previous authors $[18,19]$. However, the collected data shows that there is no significant sex predilection of regional odontodysplasia. On the contrary, other literature gives ratios as high as 1.37:1, 1.4:1 and even 1.7:1 of female to male cases, which shows a big difference from our results presented in this review (1.03:1) [18-20]. The possible explanation for this disparity can be a high number of new case reports taken into account in this article compared to older works.

It is worth noting that usually regional odontodysplasia affects quadrants or a quadrant as a whole, however, there are some examples where teeth from one quadrant and just one tooth from another one crossing the midline are affected. This was the case in a report by Courson et al. from 2003. An 11-year-old boy was diagnosed with "ghost teeth" in the upper right maxilla and the left central incisor was also affected [21]. The same was observed in a 12-year-old girl described by Carlos et al. in 2008 [22]. It is difficult to draw any concrete conclusions from this observation, but it is possible that this manifestation could indicate a different etiological factor than the rest.

In general, all of the typical known clinical symptoms were observed in the collected group. The most common, which appeared in over $90 \%$ of the patients, were yellowishbrown colour and delayed teeth eruption, but they varied in the extent. It is also possible that even more patients than stated in the table had these manifestations but were not reported by the authors either because the teeth were lost before seeking medical attention or were not noted by the clinicians. The main radiological symptom was the appearance of "ghost teeth" reported in all cases and poorly developed buds for patients with unerupted teeth $(92.2 \%)$. This finding concludes that radiographs should remain the main diagnostic method confirming RO. Local pain is reported by $44.7 \%$ of the patients. The majority do not experience any direct pain associated with the disorder. It would explain why, very often, especially in less developed countries, patients seek medical attention in later life when the teeth are already lost. The oldest patient of all the collected cases was 35 years old at the time of the diagnosis [23].

The histopathological examination of the extracted teeth shows that the most common feature is irregular calcification of dentin, followed by hypoplastic and hypomineralised enamel. It is worth pointing out that some of the examined teeth lacked the enamel layer, lost due to grinding or caries [24]. Not many, only $8.8 \%$, of the reports included dislocated cementum present on the surface of the crown. It is difficult to say whether this feature is more common for the disorder but was not reported for the same reasons as stated above or is simply rare among the affected teeth.

In our case of regional odontodysplasia, spectrometric analysis was conducted on the extracted molar. The results show a significantly lower magnesium to calcium ratio in the dysplastic tooth compared to the control healthy tooth in the exfoliation period. The same can be said about sodium to calcium ratio (data not published).

Most of the patients had characteristics of regional odontodysplasia in both dentitions, but in $28.9 \%$ only the secondary dentition was affected and in $5 \%$ only the primary. This tendency suggests that the etiological factor is present for a longer time in the majority of the cases, assuming the factor can influence the teeth in the germ formation and development period. There have been reports of only one tooth being affected and, on the other hand, generalised cases $[9,25]$. This prevalence excludes the possibility of the cause being purely systemic and implies possible etiological factors that result in a similar condition manifestation. In one of the cases an 8.5-year-old boy was diagnosed with generalised odontodysplasia [26]. All primary and secondary teeth had visible changes on the radiograph indicative of ghost teeth. The patient also suffered from other genetic disorders-dolichocephaly and clinodactyly. There was no history of trauma or other 
events that could be suspected of being the main cause of either of the conditions. This suggests there is a possibility of RO being determined genetically at least in some of the cases. Another example of generalised $\mathrm{RO}$ is a 5-year-old girl that, on the other hand, had a very significant medical history [27]. At 2.5 months old, she was diagnosed with irregular breathing problems and was operated on for a right diaphragm hernia. Two months later she was hospitalised due to an RS-virus infection. In this case, both primary and secondary dentitions were affected, the same as in the previously described one.

An important etiological factor that was mentioned several times in the literature is local trauma. In 2021, Majumdar et al. [28] described a case of a 10-year-old girl with RO of front secondary teeth in the mandible. The patient suffered a trauma to the affected region at the age of 2 months. A similar case was described in 2014 by Ramakrishnan and Menon [9]. A girl, 7 years old at the time of the diagnosis, had characteristics of regional odontodysplasia only in one tooth-the maxillary right central incisor. The girl had a history of intrusive trauma of the corresponding primary tooth, which was extracted shortly after the incident. There is one described patient, where trauma during pregnancy could have influenced the development of the condition. In a case presented by Volpato et al. [8] in 2008, a 12 year-old-female with history of being born with a partially swollen and reddish face along with a deviation of the chin to the right. The mother fell during the third trimester of pregnancy. Otherwise, she was completely healthy and there was no facial asymmetry, the only supposed consequence of the incident was "ghost teeth" in the mandibular region, crossing the midline.

The treatment of RO varies depending on the severity of the disorder. Some patients can be successfully treated by conservative restorations or root canal treatment [29], but the most common method is the extraction of the affected teeth performed in $78.6 \%$ of the patients. It is worth noting that in some cases, other possible treatment methods were applied, and in the event of no success, the extraction turned out to be necessary [26]. There are examples of patients who were treated with implantoprosthetics $(7.5 \%)$. Therefore preserving the teeth is important to promote the development of the bone to make it possible when they reach adulthood [30,31]. Regional odontodysplasia very often causes malocclusion, shift of the midline to the affected side and orthodontic treatment is then necessary $[14,32]$. As patients get older, their aesthetic expectations play a bigger role. In many cases, a partial acrylic denture is made not only to restore the correct occlusion but also to improve the appearance.

Another interesting solution is autotransplantation of tooth germs into the area, where extractions were performed, and was used in several patients with success [33-35]. The oldest case described in 1993 involved a 10-year-old girl [33]. She was referred to remove unerupted maxillary left incisors and canine due to their malformation manifested by little demarcation between enamel and dentin, as well as wide pulp chambers and root canals with open apices. After autogenous bone graft, mandibular second premolars were autotransplanted in the affected frontal area. The longest documented case of regional odontodysplasia treated by autotransplantation had a 6-year follow-up [34]. This report presented a 9-year-old girl who suffered from recurrent periapical inflammations in the mandibular right quadrant. Clinically, the causal teeth were hypoplastic with soft yellowish enamel, as well as radiographically, these teeth were manifested as "ghost teeth". The radical treatment with surgical removal of almost all teeth in the affected area under general anaesthesia was conducted. Only fully erupted incisors with $\mathrm{RO}$ were not extracted. Immediately, the first premolars from the three other quadrants were autotransplanted into the right side of the mandible. Annual postoperative radiographs showed normal condition of periodontal ligaments and alveolar bone. At the follow-up visit after 6 years, no percussion abnormalities and radiographic signs of ankylosis or external resorption of the autotransplanted teeth were observed. Thanks to the successful surgery, the symmetrical development of the mandible had been preserved. The last case with performed autotransplantation from 2012 demonstrated a boy with RO confined to the anterior mandibular region on the left side [35]. This report described the combined surgical and orthodon- 
tic treatment. In the 2-step autotransplantation, the second maxillary left premolar was transplanted in place of the removed canine and then, after a year and a half, the second maxillary right premolar was implanted in place of the removed incisors. After concomitant orthodontic treatment, a good aesthetic as well as functional effect was achieved.

Undoubtedly, the strength of our systematic review is the analysis of all case reports available in English regarding regional odontodysplasia. However, it is difficult to draw unambiguous conclusions, because the papers concern a rare disorder that is often not correctly diagnosed in everyday clinical work. Case reports are classified as having a low-evidence level and currently it is difficult to publish papers of this kind in reputable journals, which affects their reduced availability. Moreover, the case reports were published in a wide time period and have a high heterogeneity. Not all of them contained the attached full clinical picture and radiological examination, and some of them described only the diagnostic issues without performing any therapeutic methods. Additionally, the authors of the papers were not always characterised by academic affiliation, which could affect the completeness of the presented data. Despite the aforementioned limitations resulting from the individual nature of the included case reports, the above review collected and summarised the knowledge published so far about regional odontodysplasia.

\section{Conclusions}

Regional odontodysplasia is a rare developmental anomaly affecting deciduous and permanent teeth, which for several decades has been described only by authors from a few countries. Nevertheless, the radiological picture of ghost teeth accompanying clinical discolouration of tooth crowns due to enamel hypoplasia can be considered as a pathognomonic symptom. Although there is no consensus on the treatment method of choice, most cases ended with the surgical removal of the affected teeth. Similarly, the potential etiopathogenesis of this disorder has not been determined so far. It must be emphasised that patients with regional odontodysplasia require long-term specialised care due to the rapid onset and progression of the disease, as well as the gradual need to restore aesthetic and functional properties of the dentition.

Supplementary Materials: The following are available online at https:/ /www.mdpi.com/article/10 $.3390 /$ ijerph19031683/s1, the list of included case reports (in alphabetic order).

Author Contributions: Conceptualisation, K.N.; methodology, K.N.; formal analysis, K.N.; investigation and data curation, K.N. and P.W.; writing-original draft preparation, K.N. and P.W.; writing-review and editing, K.N. and A.S.; visualisation, K.N.; supervision, A.S. All authors have read and agreed to the published version of the manuscript.

Funding: This research received no external funding.

Institutional Review Board Statement: Not applicable.

Informed Consent Statement: Not applicable.

Data Availability Statement: Data are available on request from the corresponding author.

Conflicts of Interest: The authors declare no conflict of interest.

\section{References}

1. Zegarelli, E.V.; Kutscher, A.H.; Applebaum, E.; Archard, H.O. Odontodysplasia. Oral Surg Oral Med Oral Pathol 1963, 16, 187-193. [CrossRef]

2. Pandis, N.; Polido, C.; Bell, W.H. Regional Odontodysplasia. A Case Associated with Asymmetric Maxillary and Mandibular Development. Oral Surg. Oral Med. Oral Pathol. 1991, 72, 492-496. [CrossRef]

3. Cahuana, A.; González, Y.; Palma, C. Clinical Management of Regional Odontodysplasia. Pediatr. Dent. 2005, 27, 34-39. [PubMed]

4. Gomes, M.P.; Modesto, A.; Cardoso, A.S.; Hespanhol, W. Regional Odontodysplasia: Report of a Case Involving Two Separate Affected Areas. ASDC J. Dent. Child. 1999, 66, 155, 203-207. [PubMed]

5. Mathew, A.; Dauravu, L.M.; Reddy, S.N.; Kumar, K.R.; Venkataramana, V. Ghost Teeth: Regional Odontodysplasia of Maxillary First Molar Associated with Eruption Disorders in a 10-Year-Old Girl. J. Pharm. Bioallied. Sci. 2015, 7, S800-S803. [CrossRef] [PubMed] 
6. Ponranjini, V.C.; Jayachandran, S.; Bakyalakshmi, K. Regional Odontodysplasia: Report of a Case. J. Dent. Child. 2012, 79, 26-29.

7. Ozer, L.; Cetiner, S.; Ersoy, E. Regional Odontodysplasia: Report of a Case. J. Clin. Pediatr. Dent. 2004, 29, 45-48. [CrossRef]

8. Volpato, L.; Botelho, G.; Casela, L.; Borges, A.; Silva, K. Regional Odontodysplasia: Report of a Case in the Mandible Crossing the Midline. J. Contemp. Dent. Pract. 2008, 9, 142-148. [CrossRef]

9. Ramakrishnan, M.; Menon, P. Odontodysplasia Involving Single Tooth: A Rare Entity. SRM J. Res. Dent. Sci. 2014, 5, 140-142. [CrossRef]

10. Slootweg, P.J.; Meuwissen, P.R. Regional Odontodysplasia in Epidermal Nevus Syndrome. J. Oral Pathol. 1985, 14, $256-262$. [CrossRef]

11. Steiman, H.R.; Cullen, C.L.; Geist, J.R. Bilateral Mandibular Regional Odontodysplasia with Vascular Nevus. Pediatr. Dent. 1991, 13, 303-306. [PubMed]

12. Koskinen, S.; Keski-Filppula, R.; Alapulli, H.; Nieminen, P.; Anttonen, V. Familial Oligodontia and Regional Odontodysplasia Associated with a PAX9 Initiation Codon Mutation. Clin. Oral Investig. 2019, 23, 4107-4111. [CrossRef] [PubMed]

13. Fujiwara, T.; Nakano, K.; Sobue, S.; Ooshima, T. Simultaneous Occurrence of Unusual Odontodysplasia and Oligodontia in the Permanent Dentition: Report of a Case. Int. J. Paediatr. Dent. 2000, 10, 341-347. [CrossRef] [PubMed]

14. Jahanimoghadam, F.; Pishbin, L.; Rad, M. Clinical, Radiographic, and Histologic Evaluation of Regional Odontodysplasia: A Case Report with 5-Year Follow-Up. J. Dent. 2016, 17, 159-163.

15. Nijakowski, K.; Surdacka, A. Regional Odontodysplasia-A Rare Developmental Dental Anomaly. Pediatr. Pol. 2021, 96, 153-155. [CrossRef]

16. Page, M.J.; McKenzie, J.; Bossuyt, P.; Boutron, I.; Hoffmann, T.; Mulrow, C.D.; Shamseer, L.; Tetzlaff, J.; Akl, E.; Brennan, S.E.; et al. The PRISMA 2020 Statement: An Updated Guideline for Reporting Systematic Reviews. BMJ 2021, 372, n71. [CrossRef]

17. OCEBM Levels of Evidence. Available online: https://www.cebm.net/2016/05/ocebm-levels-of-evidence/ (accessed on 22 August 2020).

18. Crawford, P.J.; Aldred, M.J. Regional Odontodysplasia: A Bibliography. J. Oral Pathol. Med. 1989, 18, 251-263. [CrossRef]

19. Tervonen, S.A.; Stratmann, U.; Mokrys, K.; Reichart, P.A. Regional Odontodysplasia: A Review of the Literature and Report of Four Cases. Clin. Oral Investig. 2004, 8, 45-51. [CrossRef]

20. Alotaibi, O.; Alotaibi, G.; Alfawaz, N. Regional Odontodysplasia: An Analysis of 161 Cases from 1953 to 2017. Saudi Dent. J. 2019, 31, 306-310. [CrossRef]

21. Courson, F.; Bdeoui, F.; Danan, M.; Degrange, M.; Gogly, B. Regional Odontodysplasia: Expression of Matrix Metalloproteinases and Their Natural Inhibitors. Oral Surg. Oral Med. Oral Pathol. Oral Radiol. Endod. 2003, 95, 60-66. [CrossRef]

22. Carlos, R.; Contreras-Vidaurre, E.; de Almeida, O.P.; Silva, K.R.; Abrahão, P.G.; Miranda, A.M.M.A.; Pires, F.R. Regional Odontodysplasia: Morphological, Ultrastructural, and Immunohistochemical Features of the Affected Teeth, Connective Tissue, and Odontogenic Remnants. J. Dent. Child. 2008, 75, 144-150.

23. Miloglu, O.; Goregen, M.; Akgul, H.M.; Harorli, A. Generalized Familial Crown Resorptions in Unerupted Teeth. Eur. J. Dent. 2011, 5, 206-209. [CrossRef] [PubMed]

24. Clark, S.L.; Cairns, A.M.; Hunter, K.D. Comparative Case Report of Segmental Odontomaxillary Dysplasia and Regional Odontodysplasia. Dent. Update 2014, 41, 825-831. [CrossRef]

25. Shah, N.; Gupta, Y.K. Generalized Odontodysplasia-A Case Report. J. Indian Soc. Pedod. Prev. Dent. 1998, 16, 40-43.

26. Ibrahim Mostafa, M.; Samir Taha, N.; Ismail Mehrez, M.A. Generalised versus Regional Odontodysplasia: Diagnosis, Transitional Management, and Long-Term Followup-A Report of 2 Cases. Case Rep. Dent. 2013, 2013, 1-5. [CrossRef] [PubMed]

27. Jensen, J.L.; Ambjornsen, E.; Haanaes, H.R.; Storhaug, K. Case Report: Unclassified Syndrome Involving Dental Enamel, Dentine and Lack of Tooth Eruption. Eur. Arch. Paediatr. Dent. 2009, 10, 244-247. [CrossRef]

28. Majumdar, S.; Uppala, D.; Priyanka, K.; Kumar, S. Bilateral Regional Odontodysplasia: A Rare Case Report. Oral Maxillofac. Pathol. J. 2021, 7, 755-757.

29. Koruyucu, M.; Yaman, D.; Seymen, F.; Demirel, K.; Gençay, K. Management of Regional Odontodysplasia: A 10-Year-Follow-up Case Report and Literature Review. Eur. Oral Res. 2018, 52, 111-116. [CrossRef]

30. Abdel-Kader, M.A.; Abdelazeem, A.F.; Ahmed, N.E.-M.B.; Khalil, Y.M.; Mostafa, M.I. Oral Rehabilitation of a Case with Regional Odontodysplasia Using a Regenerative Approach-A Case Report and a Review of Literature. Spec. Care Dentist. 2019, 39, 330-339. [CrossRef]

31. Bagherpoor, M.R.; Siadat, H.; Nokar, S.; Alikhasi, M. Step-by-Step Oral Rehabilitation of a Generalized Odontodysplastic Patient with Implant-Supported Prostheses: A Clinical Report. Implant. Dent. 2010, 19, 122-127. [CrossRef]

32. Iizawa, F.; Kinjoh, N.; Taguchi, Y. Regional Odontodysplasia: Long-Term Observation of a Case on the Mandibular Left Side. Pediatric Dent. J. 2010, 20, 103-109. [CrossRef]

33. van der Wal, J.E.; Rittersma, J.; Baart, J.A.; van der Waal, I. Regional Odontodysplasia: Report of Three Cases. Int. J. Oral Maxillofac. Surg. 1993, 22, 356-358. [CrossRef]

34. von Arx, T. Autotransplantation for Treatment of Regional Odontodysplasia. Case Report with 6-Year Follow-Up. Oral Surg. Oral Med. Oral Pathol. Oral Radiol. Endod. 1998, 85, 304-307. [CrossRef]

35. Ziegler, S.; Neukam, F.W. Regional Odontodysplasia: Orthodontic Treatment and Transplantation of Premolars. Am. J. Orthod. Dentofacial. Orthop. 2012, 142, 710-719. [CrossRef] 